\title{
La canine en implantologie
}

\section{RÉSUMÉ}

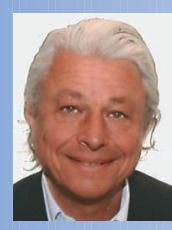

Marc BERT

D.S.O.,

Expert près la Cour d'Appel

de Paris,

1 , rue de Chazelles,

75017 Paris.
Le remplacement d'une canine par un implant va, au niveau de la neurophysiologie, remplacer un système de protection basé sur la présence de récepteurs desmodontaux par un système ankylosé dans l'os et ne transmettant que des informations faibles et filtrées aux noyaux sensitifs supérieurs. La présence ou non de récepteurs antagonistes va orienter le choix du concept occlusal à appliquer, avec comme règle première que plus le concept est simple, mieux il est réalisé. 


\section{Introduction}

$>$

Les muscles élévateurs et la plupart des

muscles abaisseurs ont leur innervation motrice commandée par le nerf trijumeau, $V^{\circ}$ paire crânienne. Cette innervation motrice est commandée par un noyau moteur dépendant lui-même d'un noyau sensitif intégrant une multitude d'informations parmi lesquelles celles issues des pressions sur les dents, pressions captées par des récepteurs situés dans le desmodonte de la dent. C'est la proprioception.

Ces «capteurs» vont induire les différentes positions de la mandibule, y compris les posi-

\section{La proprioception}

La proprioception est la fonction permettant au système nerveux central d'être renseigné sur la position d'un muscle ou d'une articulation et sur les pressions qui éventuellement s'y exercent. Dans le cas d'une dent, la proprioception renseigne les centres nerveux supérieurs sur les pressions subies par les dents, ce qui permet à ces centres de moduler la contraction des muscles manducateurs :

- soit en augmentant la pression afin d'écraser un aliment résistant ;

- soit en inhibant la pression si celle-ci dépasse des limites acceptables par l'organisme.

L'origine de la proprioception dentaire est le récepteur desmodontal situé dans le ligament alvéolo-dentaire (fig. 1). Les récepteurs desmodontaux sont :

- adaptables ou non adaptables. II s'agit soit de terminaisons libres, soit de récepteurs plus fins, encapsulés ou non ; tions pathologiques que la compréhension de l'anatomie et de la physiologie de l'ATM, des muscles et de leurs rapports vont permettre bien évidemment de traiter.

Les particularités occlusales des prothèses sur implants viennent du fait qu'il n'existe pas de récepteurs desmodontaux autour des implants qui sont ankylosés dans l'os. La canine joue un rôle majeur dans la plupart des "concepts» occlusaux par son influence sur les mouvements de la mandibule. De nombreuses questions peuvent se poser lorsqu'elle est remplacée par un implant.

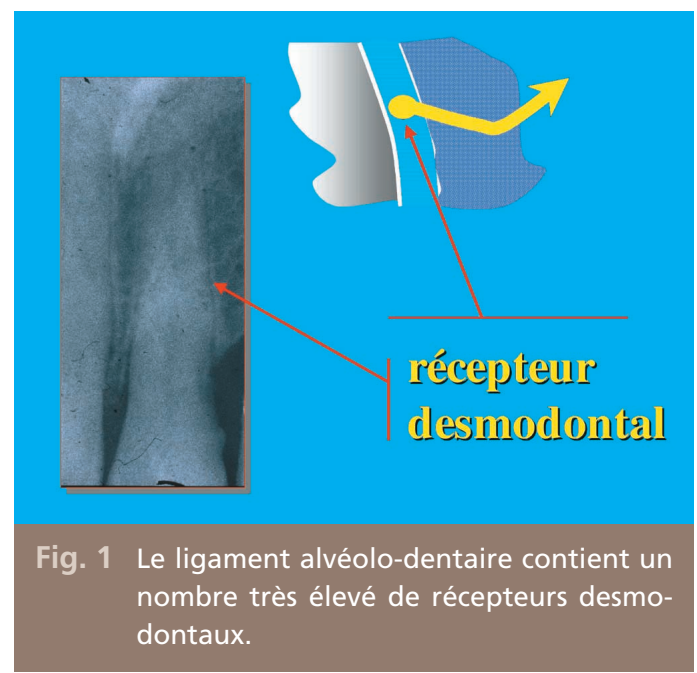

- sensibles à la vitesse d'application de la stimulation. Les forces peuvent être appliquées graduellement ou instantanément, et sont captées par des récepteurs spécifiques ;

- soumis à un seuil qui est de l'ordre de $1 \mathrm{gram}$ me (Woda, 1983) ; 
- sensibles à l'intensité de la force appliquée et capables de détecter de très faibles variations de l'amplitude de la stimulation ;

- capables d'une sensibilité directionnelle.

Les récepteurs desmodontaux sont en grand nombre pour les incisives et canines, et leur nombre va en décroissant des prémolaires aux molaires. Le récepteur desmodontal enregistre les pressions exercées sur une dent, qu'elles soient axiales ou latérales, et transmet ces pressions au noyau sensitif du trijumeau (cinquième paire crânienne ou V) par l'intermédiaire d'un premier neurone (ou protoneurone) dont le corps cellulaire se situe dans le ganglion de Gasser (fig. 2). L'information circule :

- par la branche maxillaire du trijumeau, ou V2, pour les dents maxillaires ;

- par la branche mandibulaire du trijumeau, ou V3, pour les dents de l'arcade inférieure.

La branche V1, l'opthalmique de Willis, ne transmet aucune information d'origine dentaire.

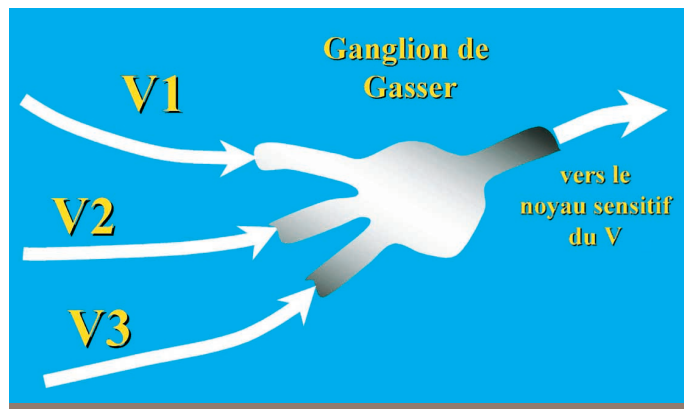

Fig. 2 Les informations captées par les récepteurs desmodontaux maxillaires et mandibulaires passent par le ganglion de Gasser (siège du corps cellulaire des neurones) et arrivent sur le noyau sensitif du trijumeau.
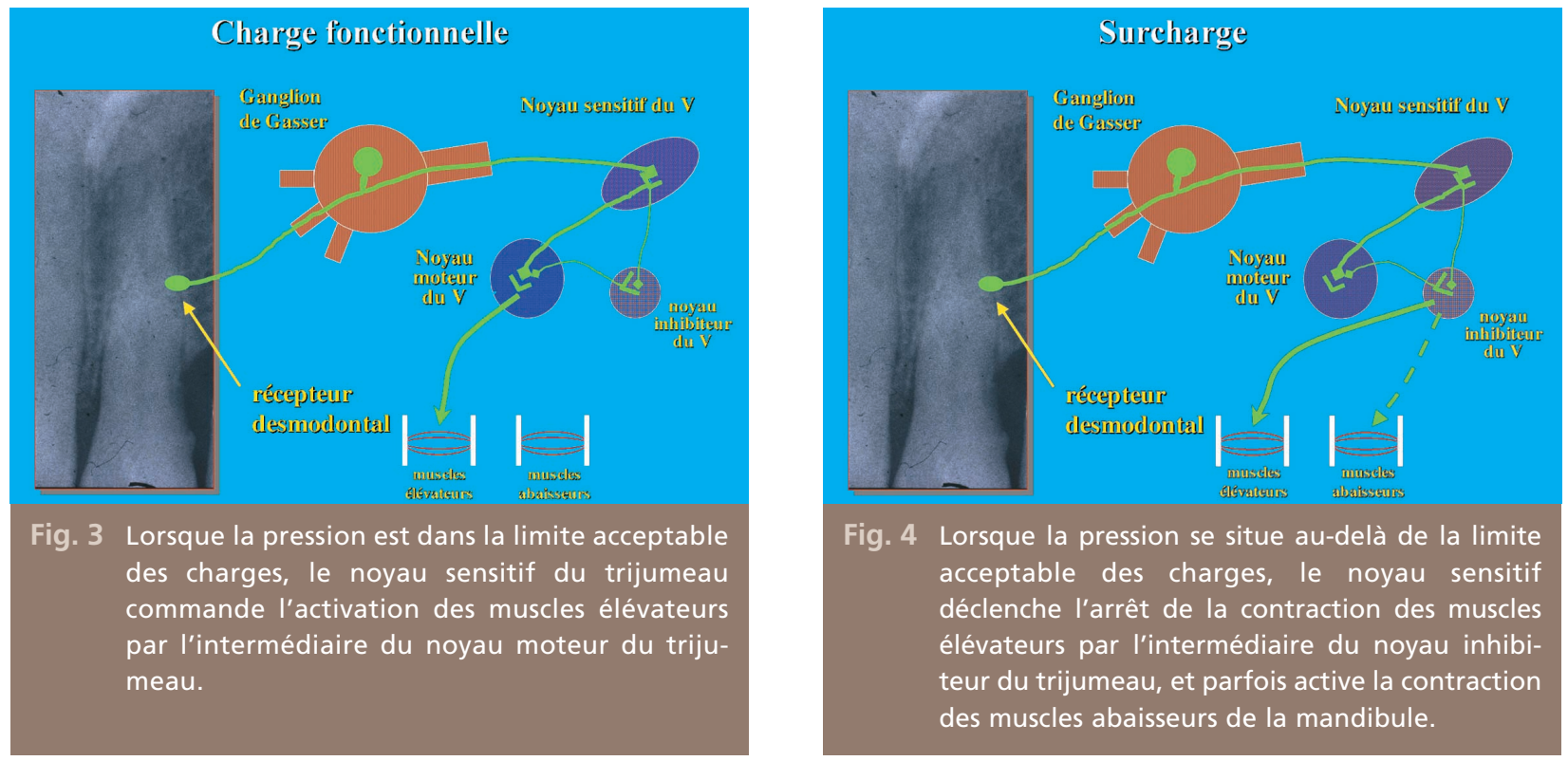
En simplifiant à l'extrême, l'information perçue par le noyau sensitif du trijumeau est transmise à 2 noyaux différents, en fonction de l'intensité des pressions enregistrées par le récepteur desmodontal :

- le noyau moteur du $V$, qui déclenche la contraction des muscles élévateurs pour augmenter leur action afin d'écraser un aliment (fig. 3) ;

- le noyau inhibiteur du V, si la pression dépasse certaines limites physiologiques (fig. 4). La contraction musculaire est alors stoppée, avec parfois contraction des muscles abaisseurs en cas de choc sur la dent.

Le seuil d'apparition d'une réponse à une stimulation mécanique est très bas et a été estimé à 1 gramme par Woda et Fontenelle (1975) et Woda (1983). Cette sensibilité a été qualifiée par ces auteurs d'épicritique et de directionnelle. Une sensibilité épicritique est fine et transmise par des fibres nerveuses de gros calibre, très myélinisées et ne perdant aucune information.

La sensibilité épicritique d'origine desmodontale est directionnelle car le patient testé est capable d'indiquer si la force appliquée l'est en pression ou en traction, confirmant ainsi sa finesse de perception.

En résumé, le parodonte est à l'origine d'une activité neuro-physiologique très fine et très discriminative, mettant en jeu un mécanisme protecteur très organisé, à partir d'un seuil très bas.

\section{Les «concepts» occlusaux}

La reconstruction d'une bouche s'appuie sur des conceptions permettant aux articulations temporo-mandibulaires et aux muscles qui l'actionnent de "fonctionner» dans une enveloppe physiologique de mouvements.

\section{En position \\ d'intercuspidie maximale}

Trois principes vont guider la reconstruction prothétique en position d'intercuspidie maximale :

1 - trouver ou retrouver une position d'intercuspidie avec une absence de contraction des muscles ptérygoïdiens latéraux ;

2 - trouver ou retrouver des courbes de Spee et de Wilson correctes ;

3 - obtenir une stabilité des contacts.

\section{En propulsion}

Lors de la propulsion, le glissement des incisives inférieures sur la face palatine des incisives maxillaires doit entraîner instantanément

\section{Propulsion

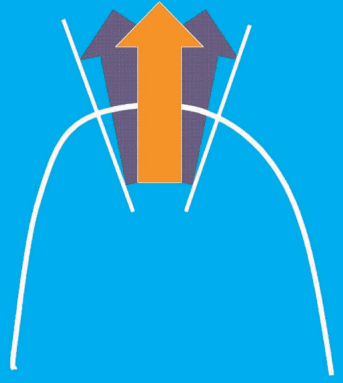

Fig. 5 Le mouvement de propulsion présente une faible amplitude en latéralité, facilitant en clinique le réglage de ce mouvement. 
la désocclusion des secteurs cuspidés. C'est le «guide antérieur». Le réglage du guide antérieur est facilité par la faible amplitude en latéralité du mouvement de propulsion (fig. 5), permettant d'obtenir un guidage satisfaisant dans l'ensemble des mouvements de glissement antérieur.

\section{En latéralité}

Plusieurs conceptions de la latéralité ont été proposées: fonction de groupe, occlusion «balancée» et fonction canine; conceptions qu'ils faut apprécier à l'aune de leur intérêt théorique et de leur "réalisibilité», c'est-à-dire de leur application correcte en bouche :

1 - La fonction de groupe. En théorie, c'est le concept idéal, plusieurs groupes de dents assurant le guidage de la mandibule du côté travaillant, répartissant les charges sur plusieurs éléments au lieu d'un seul. Au niveau pratique, il existe cependant quelques écueils :

- il n'existe pas une seule latéralité, mais une infinité de latéralités (fig. 6). Vouloir,

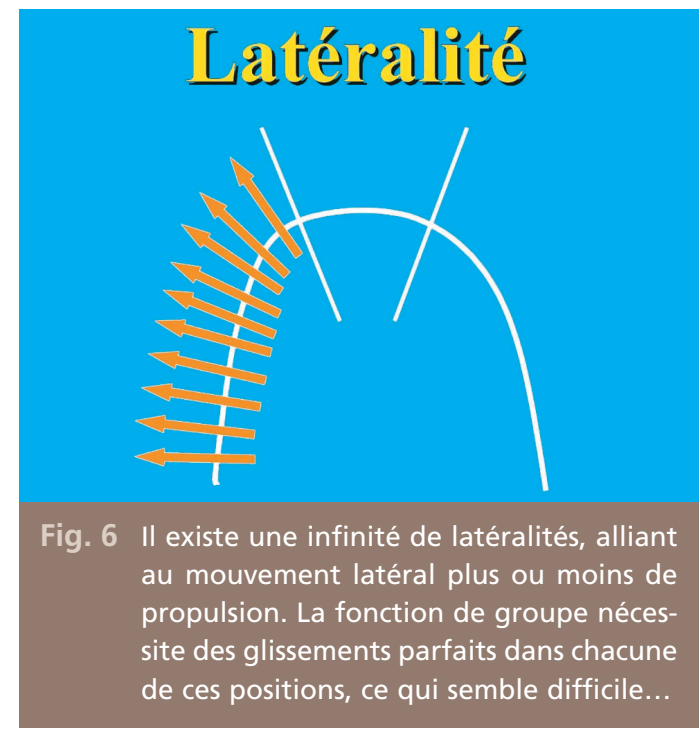

dans chacun de ces mouvements, obtenir des contacts fonctionnels est extrêmement difficile, voire impossible, en sachant qu'une erreur d'un degré sur une pente cuspidienne peut transformer un contact stabilisant en interférence. De plus, le stress accumulé au cours de la journée fait que la position de la mandibule n'est pas la même le matin et le soir, compliquant encore plus la situation (fig. 7 à 9) ;

- cette conception oblige à raccourcir la canine (fig. 10), ce qui a un impact non négligeable sur l'esthétique du sourire, d'autant plus si la canine controlatérale conserve une longueur normale (fig. 11, 12).

2 - La fonction balancée. Cette conception, mise au point en prothèse adjointe complète, permet d'obtenir la stabilisation de prothèses amovibles totales dans toutes les excursions mandibulaires. Dans le mouvement de propulsion, il existe des contacts postérieurs afin de stabiliser la prothèse maxillaire et éviter sa désinsertion postérieure. En latéralité, on construit une fonction de groupe du côté travaillant et on conserve un ou des glissements stabilisants du côté non travaillant, toujours afin d'éviter le basculement des prothèses amovibles. L'obtention de ces contacts est difficile à obtenir (et souvent l'oeuvre de spécialistes), les défauts de construction (au niveau des pentes cuspidiennes) étant compensés par la dépressibilité de la muqueuse qui permet une marge d'erreur importante. La transposition de cette conception de l'occlusion en prothèse fixe est illusoire car il n'existe aucune marge d'erreur possible, en particulier les contacts stabilisants du côté non travaillant sont le plus souvent transformés en interférences, particulièrement destructrices. 


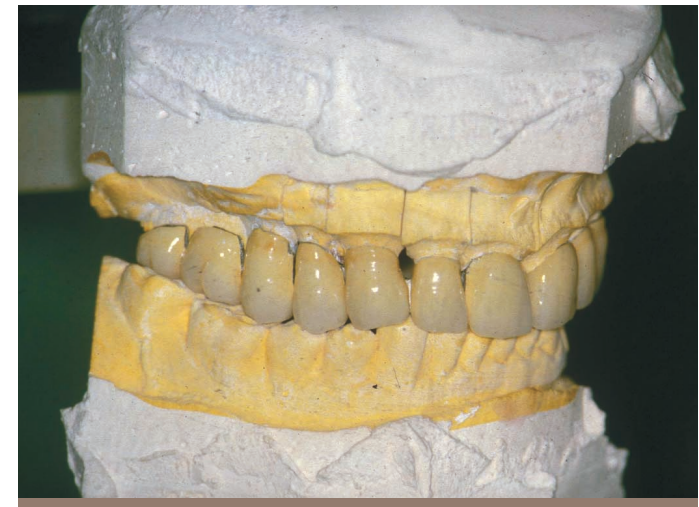

Fig. 7 Un articulateur correctement programmé permet d'obtenir des contacts en glissement sur plusieurs groupes de dents.

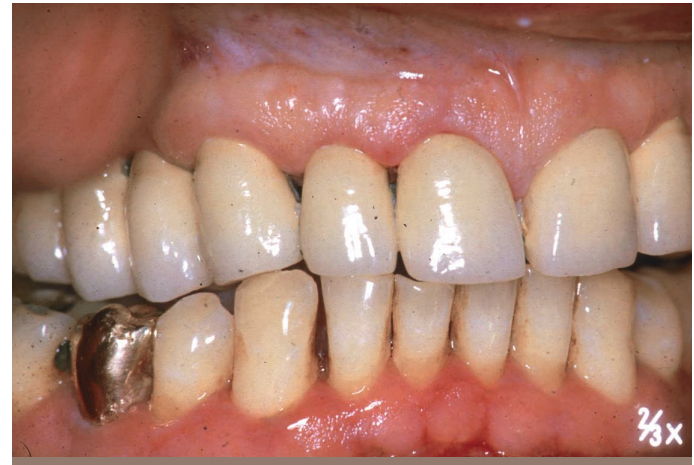

Fig. 8 En bouche, le contrôle effectué un matin montre que des contacts de groupe en latéralité peuvent être obtenus.

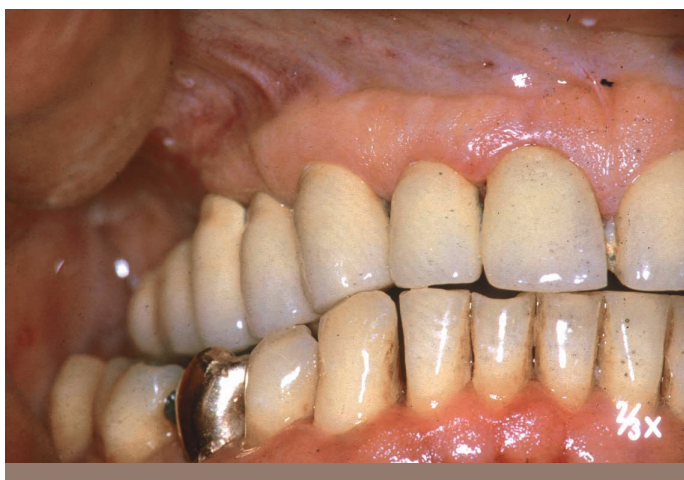

Fig. 9 Par contre, le soir, la position de la mandibule n'est plus la même et la trajectoire en latéralité est totalement modifiée.

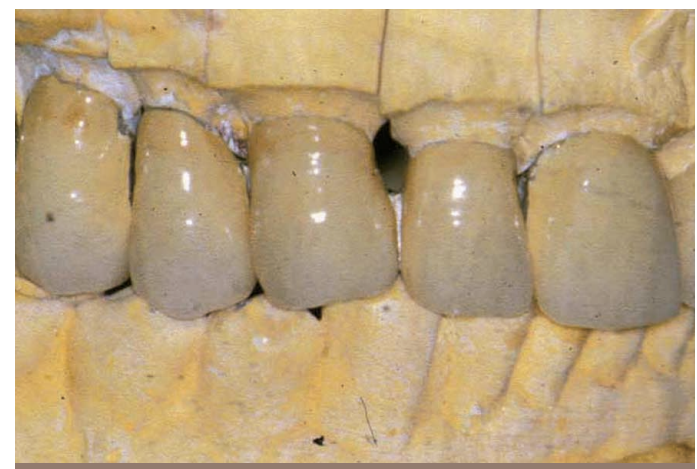

Fig. 10 Un gros plan de la figure 7 montre que la canine doit être raccourcie pour permettre la fonction de groupe, ce qui est esthétiquement très discutable, mais acceptable dans ce cas car les 2 canines ont subi le même traitement.

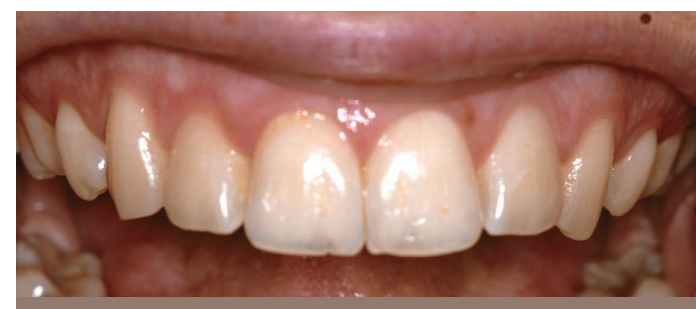

Fig. 11 Le sourire montre, dans ce cas, des canines de bonne longueur et équilibrées.

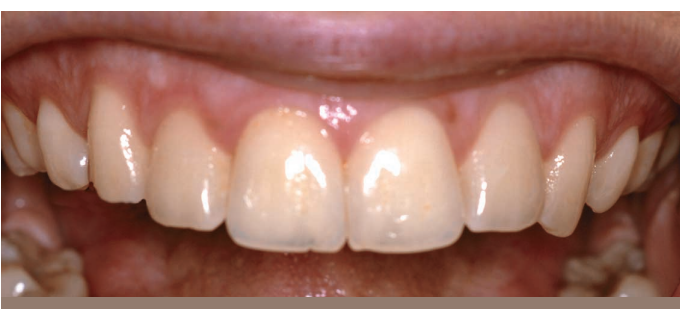

Fig. 12 La canine droite a été retouchée (merci Photoshop...) afin de permettre une fonction de groupe du côté droit, rendant inesthétique le sourire par la différence de longueur des 2 canines. 
3 - La fonction canine. Dans ce concept, le mouvement de latéralité entraîne une désocclusion immédiate des groupes cuspidés, du côté travaillant et du côté non travaillant par le glissement de la canine inférieure sur la face palatine de la canine supérieure (fig. 13, 14). Ce concept simple ne fait intervenir que 4 dents, les canines dont on a vu qu'elles étaient très richement dotées en récepteurs desmodontaux, permettant de moduler la contraction des muscles manducateurs. Un articulateur simple permet de construire facilement ce

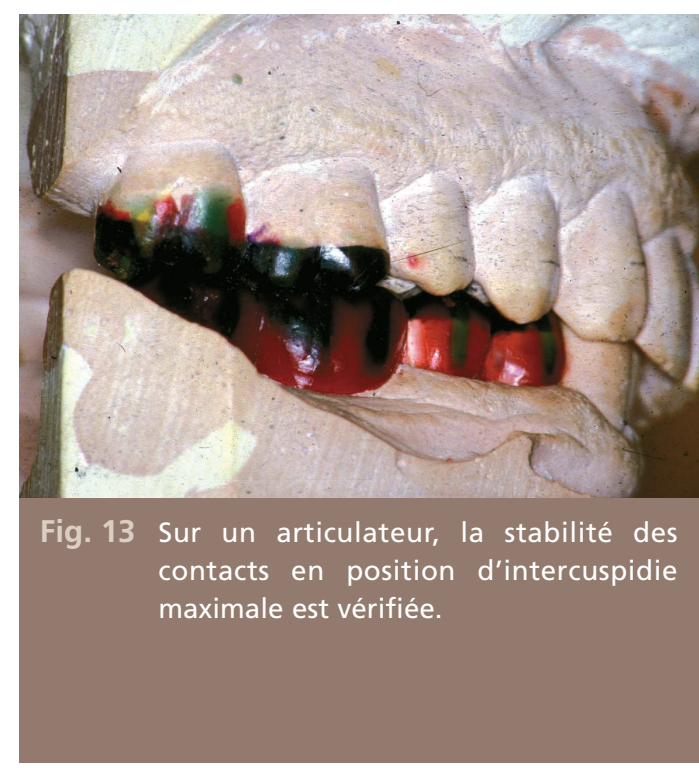

glissement qui est vérifié en bouche avec les prothèses d'attente, puis avec les prothèses d'usage.

D'une manière routinière, le concept de la fonction canine permet de construire des prothèses fonctionnelles, simples (donc bien réalisées...) et bénéficiant de la sécurité apportée par la présence de récepteurs desmodontaux en grand nombre sur la canine, permettant la modulation de la contraction de la musculature manducatrice et évitant ainsi les surcharges occlusales destructrices pour les dents, le parodonte ou les articulations temporo-mandibulaires.

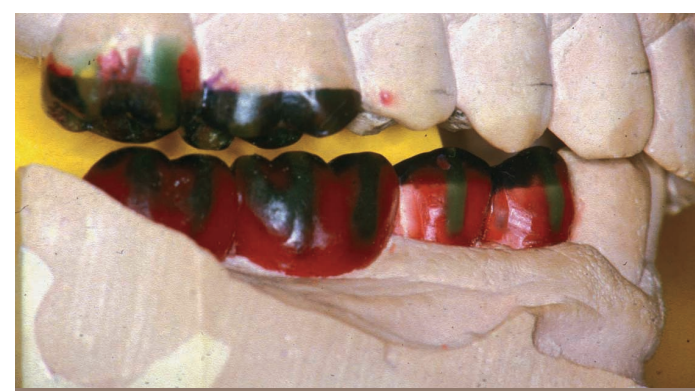

Fig. 14 En latéralité, le glissement de la canine inférieure sur la face palatine de la canine supérieure permet d'obtenir une désocclusion immédiate des groupes cuspidés, du côté travaillant et du côté non travaillant.

\section{Neurophysiologie d'un implant dentaire}

On l'a vu plus haut, les récepteurs desmodontaux d'une dent sont sensibles à des pressions dont le seuil est d'un gramme, et donnent des informations épicritiques (fines, discriminatives, véhiculées par des fibres de gros diamètre très myélinisées) et directionnelles (la direction du stimulus est perçue).
Qu'en est-il au niveau d'un implant dentaire (fig. 15) ?

En d'autres termes, quel est le devenir du récepteur desmodontal et de son protoneurone après l'extraction d'une dent ? Est-il totalement détruit? Se transforme-t-il en une terminaison nerveuse plus simple: fibre ultra- 


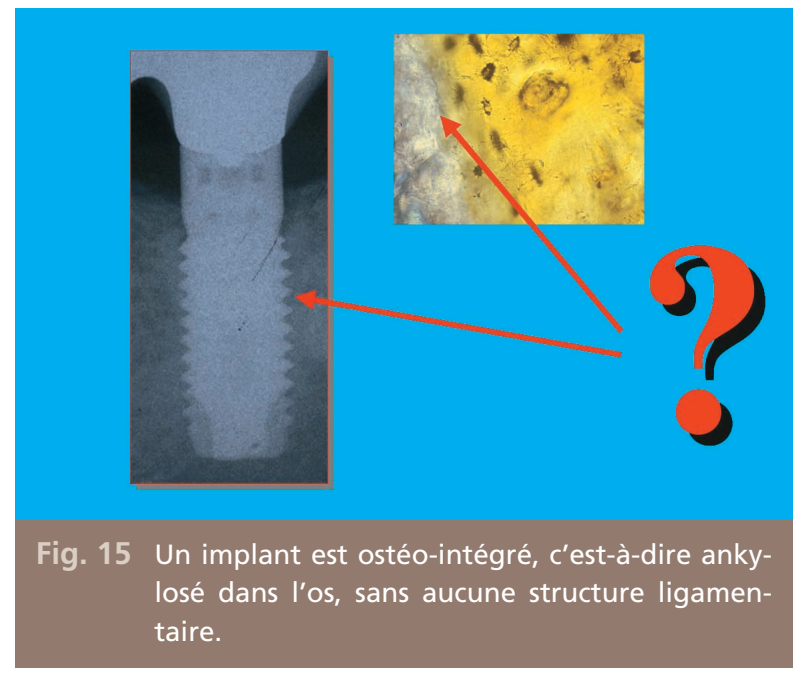

terminale, terminaison libre (nociceptive) ? Est-il intégralement conservé ?

\section{Expérimentation clinique}

L'expérimentation clinique simple permet d'apporter des réponses à ces interrogations.

À partir de 1981, et publiée en 1987 (Bert, 1987), une étude a été menée sur 50 implants de type lame de Linkow ou vis de Cherchève, c'est-à-dire non-enfouissables, donc fibro-intégrés. Le patient est initialement «éduqué» sur une dent naturelle présente en bouche, lui permettant de comprendre les informations attendues de lui. Un dynamomètre sensible au gramme (fig. 16, 17) est connecté à une dent par l'intermédiaire d'un fil de soie. Le seuil de 1 gramme est généralement retrouvé sur cette dent. Le même dispositif est ensuite placé sur un implant, et le seuil est de 8 grammes. La même expérimentation a été reprise à partir de 1991 sur des implants ostéo-intégrés, et des résultats identiques ont été trouvés: un seuil de 8 grammes en moyenne. Mais dans tous les
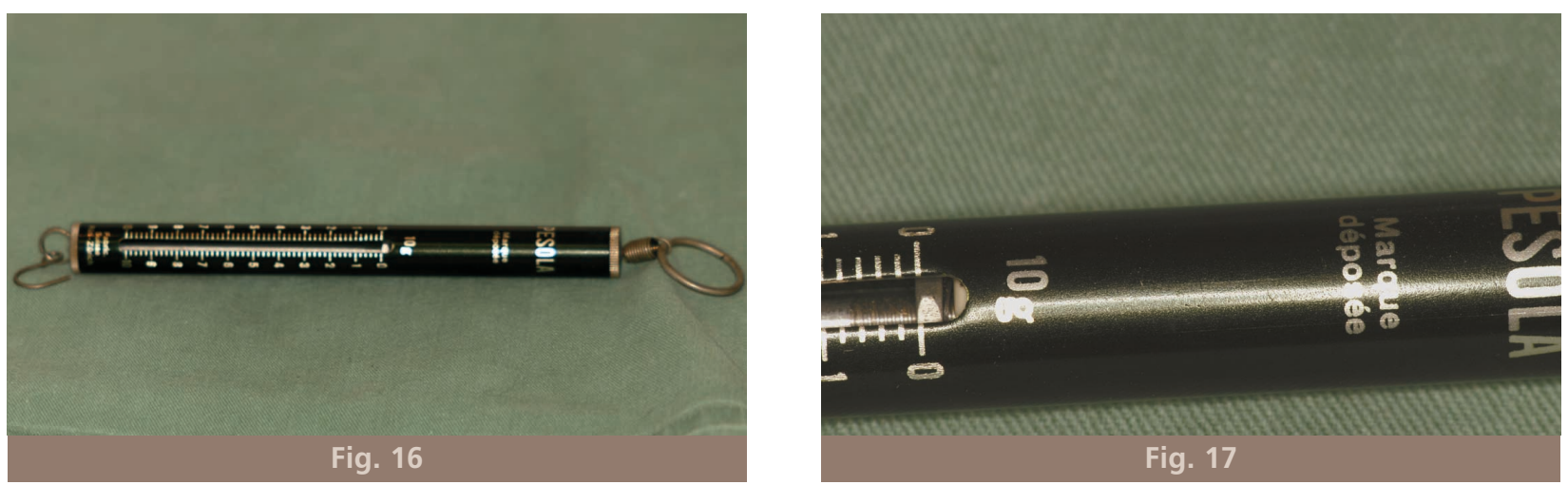

Fig. 16, 17 Un dynamomètre sensible au gramme est connecté à une dent, puis à un implant à l'aide d'un fil de soie. 

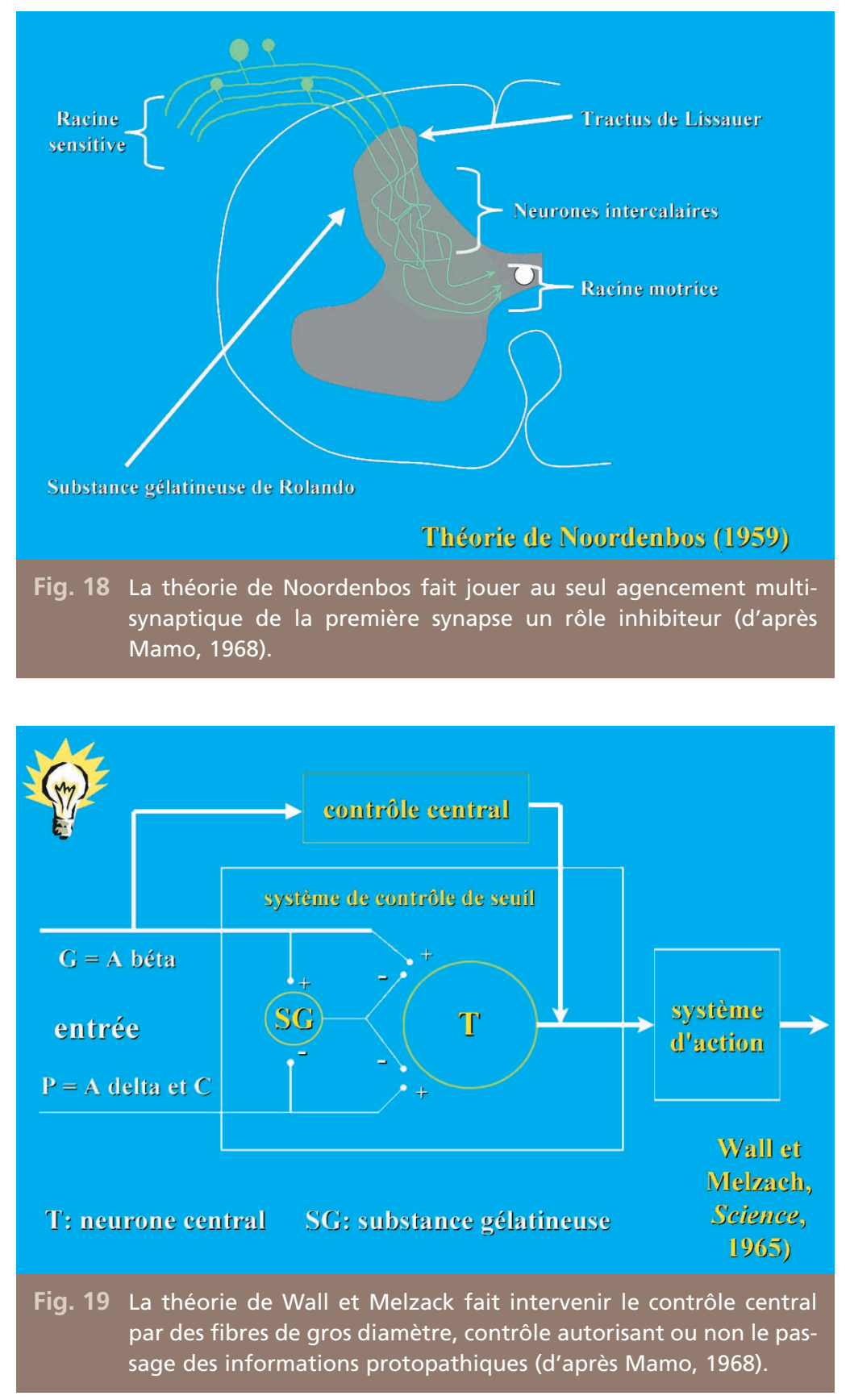

cas, la sensation a été décrite par les patients comme étant vague, diffuse, ce que les physiologistes qualifient comme étant «protopathique», c'est-à-dire véhiculée, comme pour la douleur diffuse, par des fibres de faible calibre, pas ou peu myélinisées, et surtout soumises à une filtration. Plusieurs théories ont été émises pour expliquer cette filtration: celle de Noordenbos, au niveau de la première synapse (fig. 18), ou celle actuellement plus en vogue du "gate control» de Wall et Melzack (fig. 19).

Quelle que soit la théorie retenue, il est fondamental de comprendre que les informations 
reçues par les structures péri-implantaires semblent être véhiculées par des voies qui sont soumises à un mécanisme de régulation au niveau de leur première synapse, alors que les afférences desmodontales ne sont pas soumises à ce mécanisme. Et si toutes les informations desmodontales, même les plus minimes, déclenchent un mécanisme protecteur qu'un praticien «occluso-conscient» saura détecter, il n'en est pas de même avec les implants dentaires, car seules les informations les plus importantes sont acheminées aux centres supérieurs.

\section{Conséquences cliniques}

La surcharge occlusale sur une dent déclenche tout le mécanisme protecteur décrit plus haut. L'absence de proprioception sur un implant ne met pas en jeu ce mécanisme, et la surcharge se maintient. Selon les lois de Wollf (1892), la réponse osseuse va dépendre de l'intensité de la charge: renforcement de I'os si elle est dans les limites physiologiques, destruction de l'os lorsqu'elle dépasse ces limites physiologiques (fig. 20). Parfois, la sur- charge occlusale entraîne la fracture d'un ou de plusieurs implants, de vis de prothèse, d'armatures métalliques et/ou de prothèses antagonistes.

Cette volonté de protéger l'implant de toute surcharge occlusale a conduit de nombreux auteurs, peu "occluso-conscients» et mal informés sur la physiologie, à émettre des notions hélas répandues sur les concepts occlusaux à adopter sur les implants dentaires, comme la

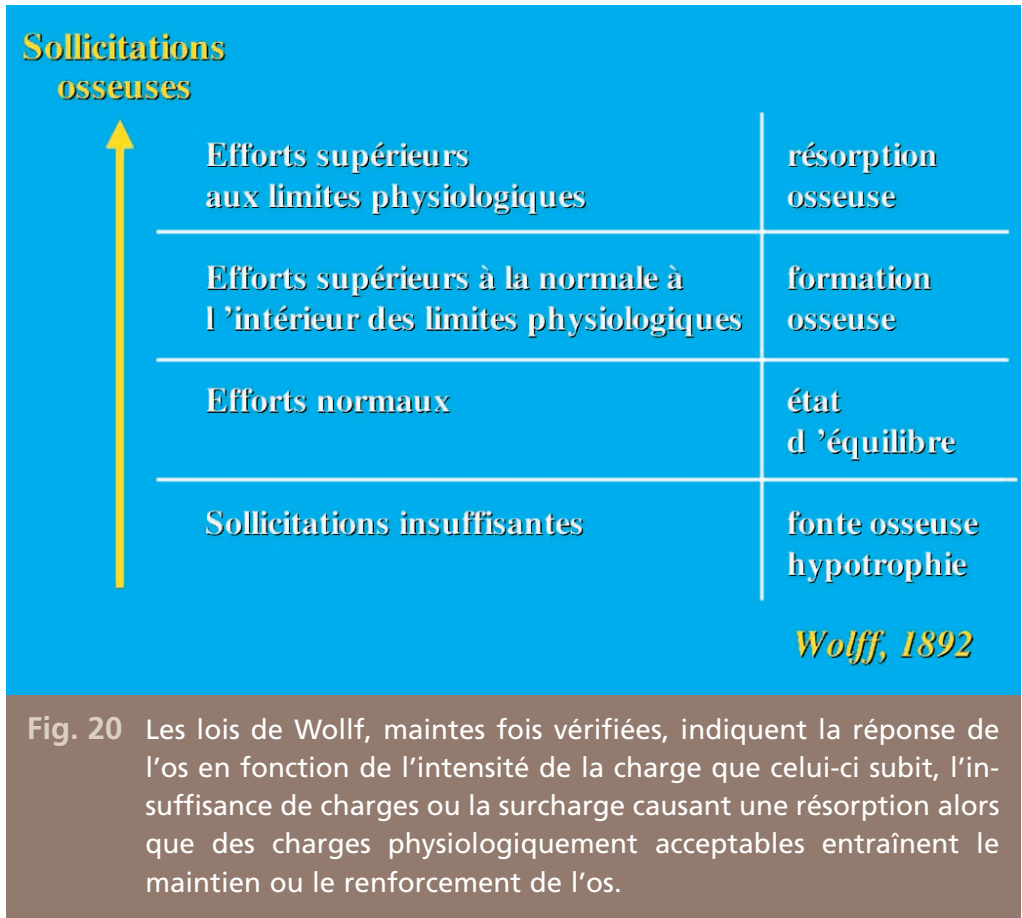


mise systématique des prothèses en sous-occlusion ou la fonction de groupe :

- la mise en sous-occlusion. II a souvent été proposé de mettre la couronne sur implant en «légère sous-occlusion», ou bien de n'autoriser les contacts sur un implant qu'en occlusion serrée du fait de l'absence de résilience d'un implant par rapport à une dent. Ces propositions traduisent une méconnaissance totale de la physiologie d'une dent. À partir du moment où une dent est en sousocclusion, elle subit une égression physiologique lui permettant de retrouver un calage occlusal nécessaire à sa stabilité. Et ce calage occlusal maintient la dent en place à chaque intercuspidie liée par exemple à la déglutition à vide (1 500 fois par jour !), l'empêchant d'égresser. Si l'implant est mis en sous-occlusion, la dent antagoniste va égresser afin de retrouver son nécessaire calage occlusal, avec un rapport qui n'a pas été choisi par le praticien et qui peut s'avérer être incorrect ;

- la fonction de groupe. Théoriquement idéale, la fonction de groupe permet, en latéralité, de répartir les contraintes occlusales sur un

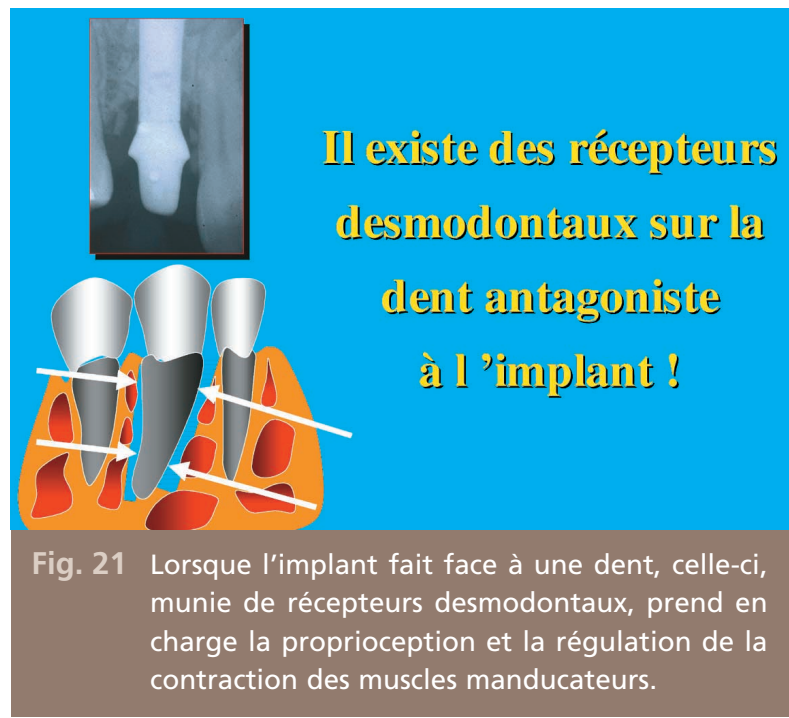

nombre important de dents. On l'a vu plus haut, une fonction de groupe correcte est très difficile à réaliser, pour des raisons techniques, physiologiques et esthétiques.

La plupart des auteurs prônant la fonction de groupe lorsque la canine est un implant ont oublié que l'occlusion est le rapport entre deux arcades dentaires, l'arcade maxillaire et l'arcade mandibulaire. Lorsqu'un implant est en occlusion, il peut être face à :

- une dent, munie d'un desmodonte et de récepteurs desmodontaux, permettant au mécanisme neurophysiologique de protection d'être présent et d'éviter les surcharges occlusales (fig. 21) ;

- une selle prothétique, ce sont alors les récepteurs de la muqueuse buccale, fins et discriminatifs, qui prennent le relais des récepteurs desmodontaux et permettent la mise en œuvre de la protection neurophysiologique (fig. 22) ;

- un implant, et dans cette situation, il n'existe aucun mécanisme protecteur permettant d'éviter les surcharges occlusales (fig. 23).

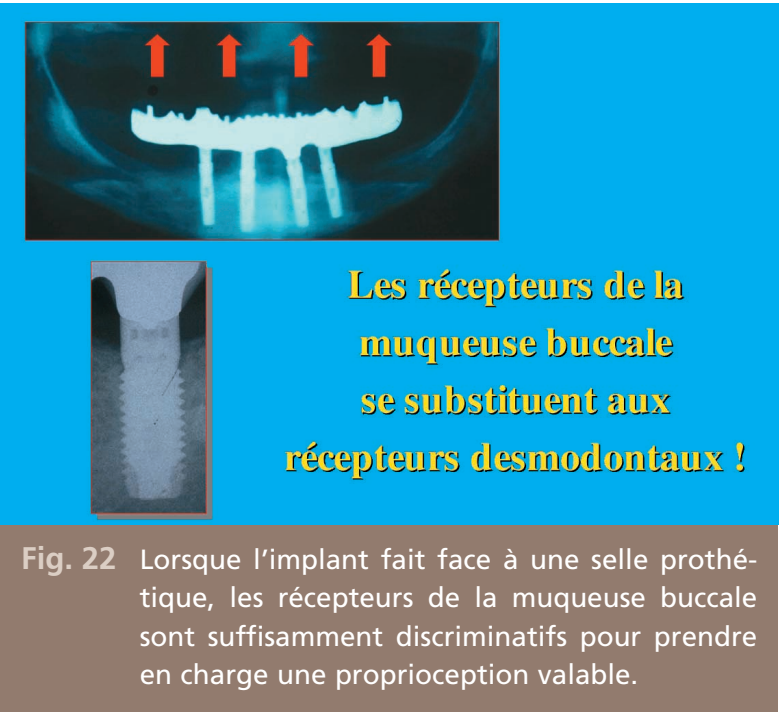




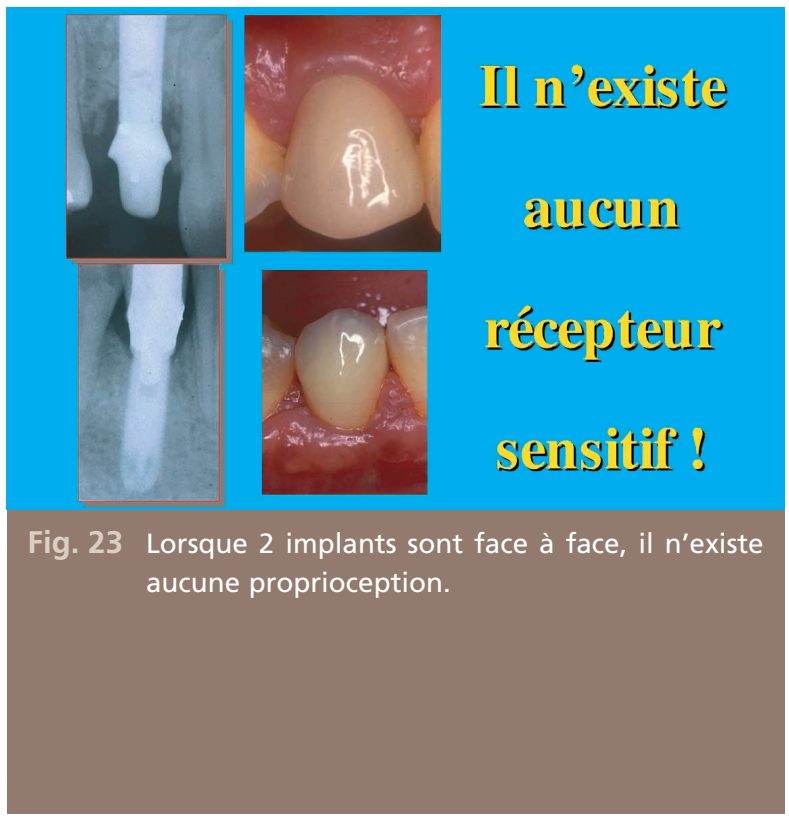

Le concept occluso-prothétique adopté doit être déterminé en fonction de l'absence ou de la présence de récepteurs desmodontaux, les seuls permettant de moduler la contraction de

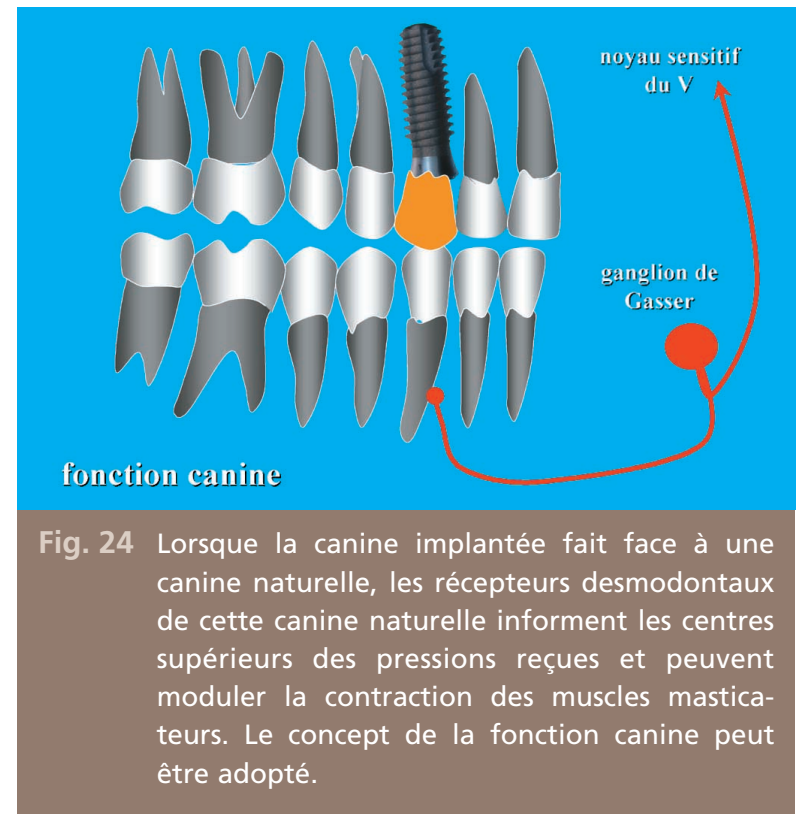

la musculature manducatrice et d'éviter les surcharges occlusales. Lorsque l'implant remplace une canine, la nature de l'arcade antagoniste devient essentielle :

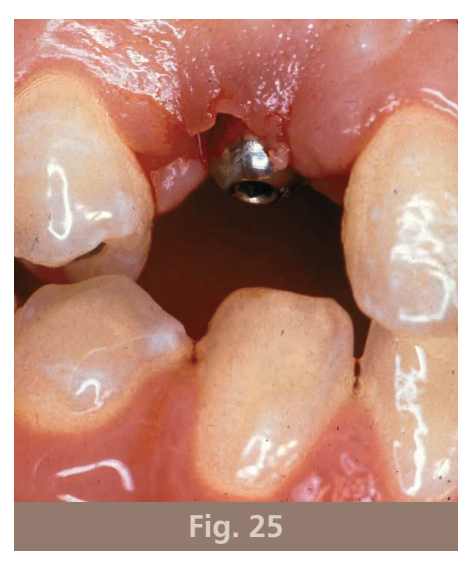

Fig. 25, 26, 27

La canine maxillaire, absente, est remplacée par un implant et fait face à une canine naturelle munie de récepteurs desmodontaux. La canine implantée participe à la propulsion et permet l'organisation d'une fonction canine.
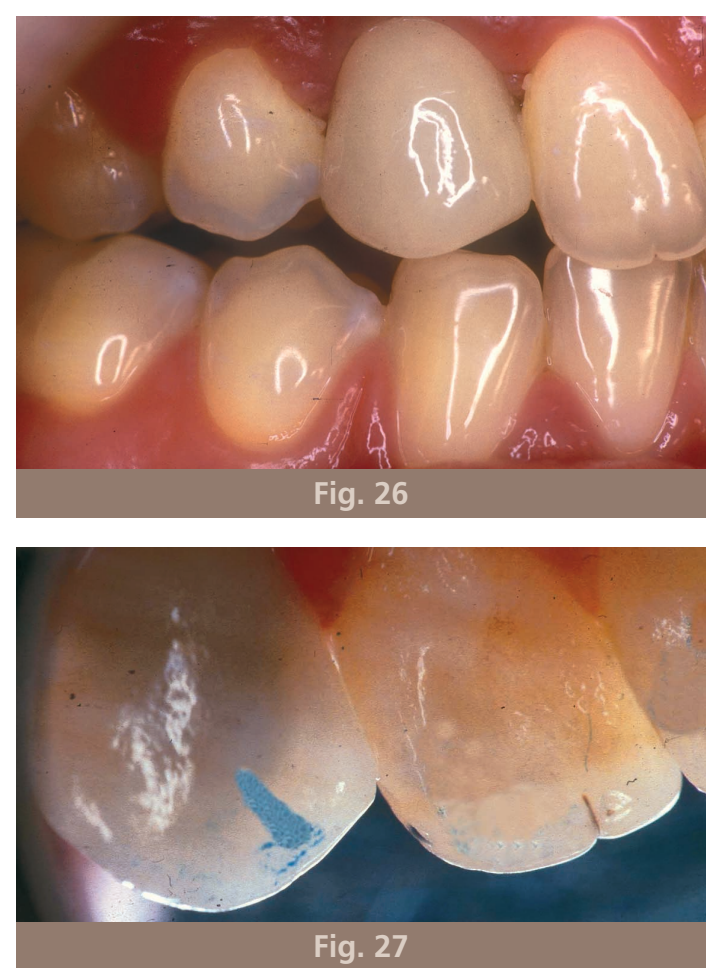
- l'élément antagoniste est une canine naturelle : c'est la meilleure situation possible, car ce sont les récepteurs desmodontaux de la canine antagoniste, qu'elle soit pulpée ou non, porteuse d'une prothèse ou non, qui capteront l'éventuelle interférence et induiront la réponse protectrice des centres supérieurs (fig. 24). Une fonction canine impliquant un implant est alors parfaitement acceptable, la dent antagoniste permettant une protection d'origine neurophysiologique (fig. 25, 26, 27), (fig. 28, 29, 30) ;

- l'élément antagoniste est un implant: cette situation clinique est rare et n'a été rencontrée qu'une fois par l'auteur en plus de 35 années d'expérience, et doit être analysée logiquement (fig. 31). La position d'intercuspidie maximale et la propulsion seront trai- tées classiquement car elles font intervenir, en plus des implants, des dents naturelles et leurs récepteurs desmodontaux. La latéralité travaillante faisant intervenir 2 implants antagonistes doit bénéficier d'une fonction de groupe «réduite», impliquant les dents bordant l'édentement: incisive latérale et première prémolaire au maxillaire et à la mandibule, avec une réduction de la hauteur des canines (fig. 32). Afin de pallier la difficulté de réalisation d'une fonction de groupe correcte, incluant toutes les positions possibles en latéralité, il faut adopter le concept de la désocclusion retardée des secteurs cuspidés proposée, entre autres, par Preiskkel dans les années 1980. Selon ce concept, les premiers millimètres de la trajectoire, enveloppe habituelle de la majorité des mouve-
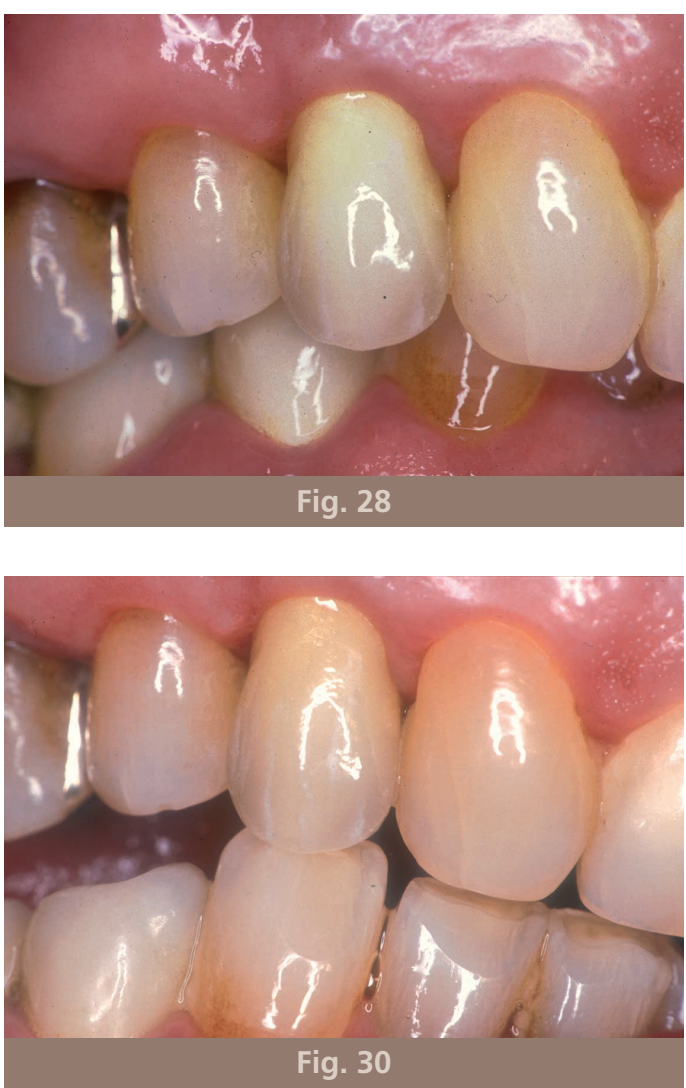

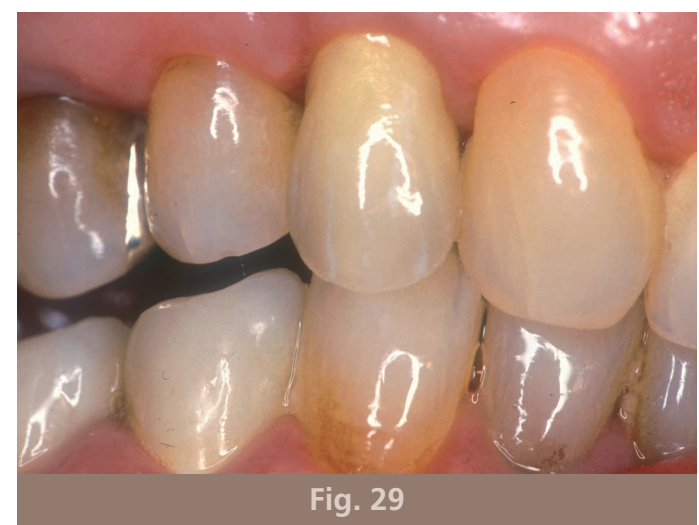

Fig. 28, 29, 30

Là encore, la canine sur implant faisant face à une canine naturelle, le concept de la fonction canine peut être appliqué, y compris lors des mouvements les plus extrêmes. 
ments occlusaux, se font en fonction de groupe, puis, lorsque le mouvement s'amplifie et sort de son enveloppe habituelle, intervient une fonction canine. Ce concept per-
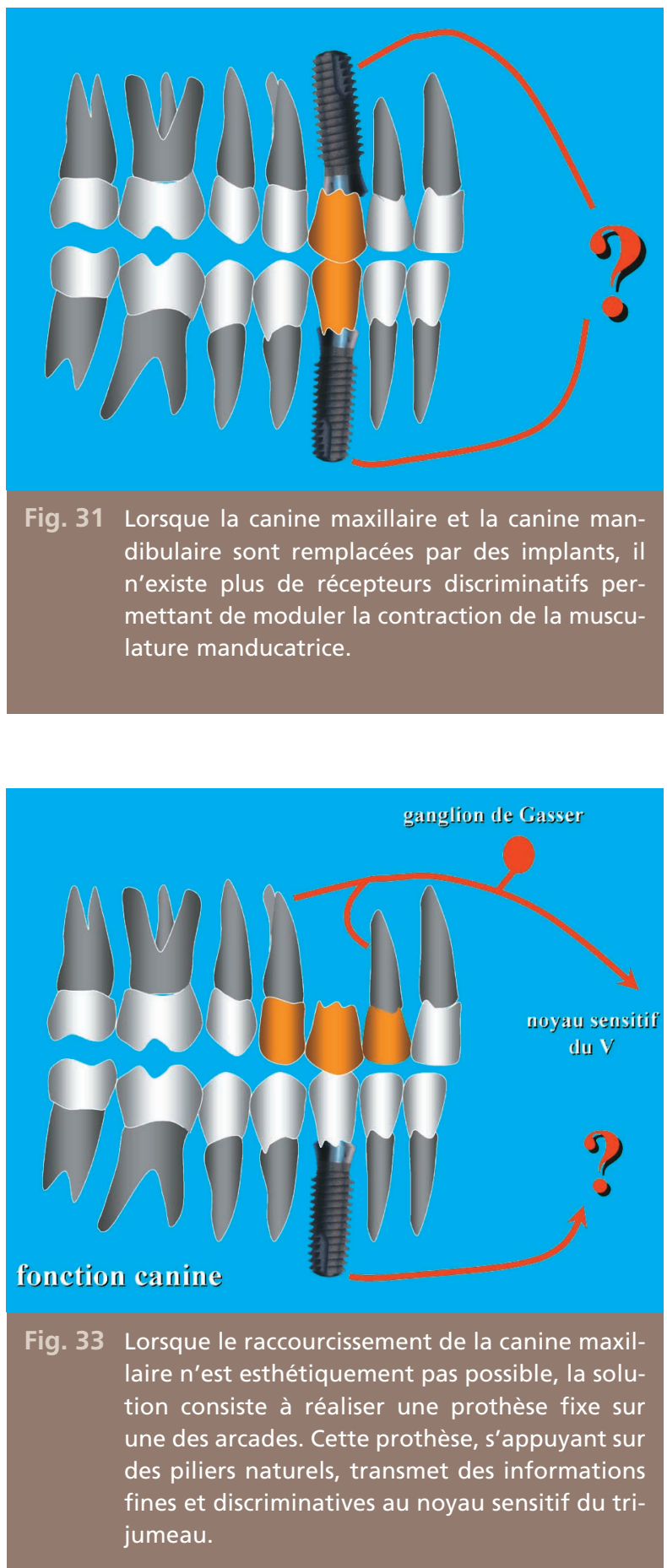

met de limiter le réglage des plans de glissement en latéralité aux premiers millimètres du mouvement, réduisant ainsi la difficulté de leur réalisation, puis, lorsque le mouve-
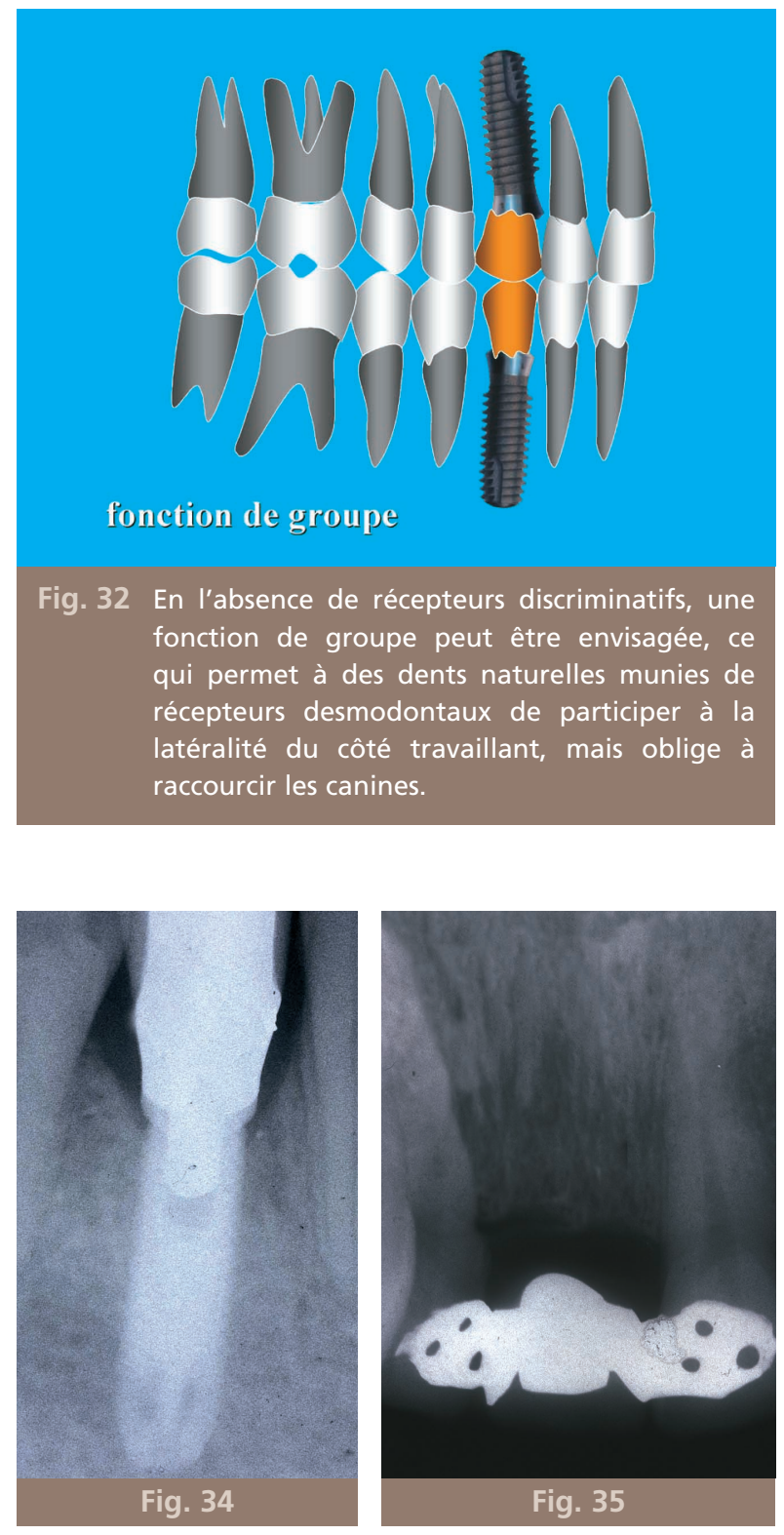

\section{Fig. 34, 35}

Dans ce cas unique de perte des canines maxillaire et mandibulaire droites, et la canine maxillaire gauche étant de longueur importante, l'option clinique choisie a fait intervenir un implant à la mandibule et un bridge collé au maxillaire. 
ment s'amplifie, ce qui est en dehors de l'enveloppe habituelle des mouvements, donc rare, une fonction canine prend le relais. II faut cependant bien noter que, même avec une désocclusion retardée et une fonction de groupe réduite à quelques dents, le raccourcissement de la canine maxillaire est indispensable, avec les conséquences esthétiques vues au paragraphe précédent. Le patient doit impérativement en être informé et, en cas de refus ou lorsque la canine contro-latérale est d'une grande longueur, l'indication de I'implant maxillaire doit être remise en cause au profit d'un bridge impliquant la préparation pelliculaire pour un bridge collé ou périphérique des dents collatérales pour un bridge classique (fig. 33, 34, 35).

L'intermédiaire de prothèse remplaçant la canine maxillaire prend appui sur les dents naturelles bordant l'édentement, dents munies de récepteurs desmodontaux et permettant alors de moduler la contraction des muscles manducateurs.

En clinique, lorsque des canines sont absentes et doivent être remplacées par des implants, la recherche des récepteurs desmodontaux permet de s'orienter vers un concept occlusal réfléchi, avec à chaque fois, comme idée maîtresse, que le concept le plus simple sera le mieux réalisé (fig. 36, 37, 38).

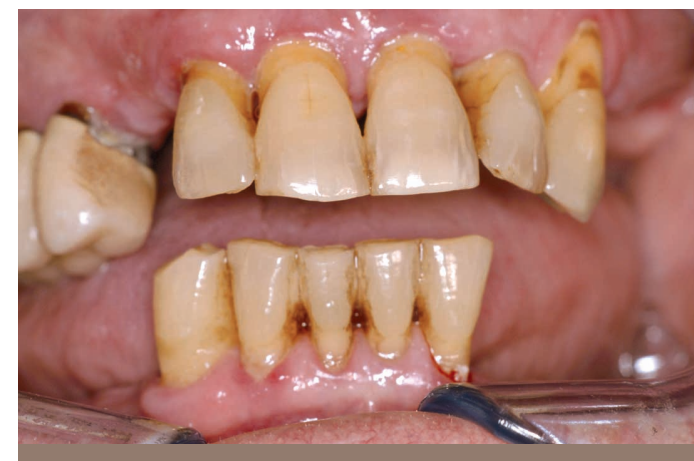

Fig. 36 L'examen clinique montre la perte de la canine maxillaire droite et de la canine mandibulaire gauche. La canine maxillaire gauche et la canine mandibulaire droite sont présentes.
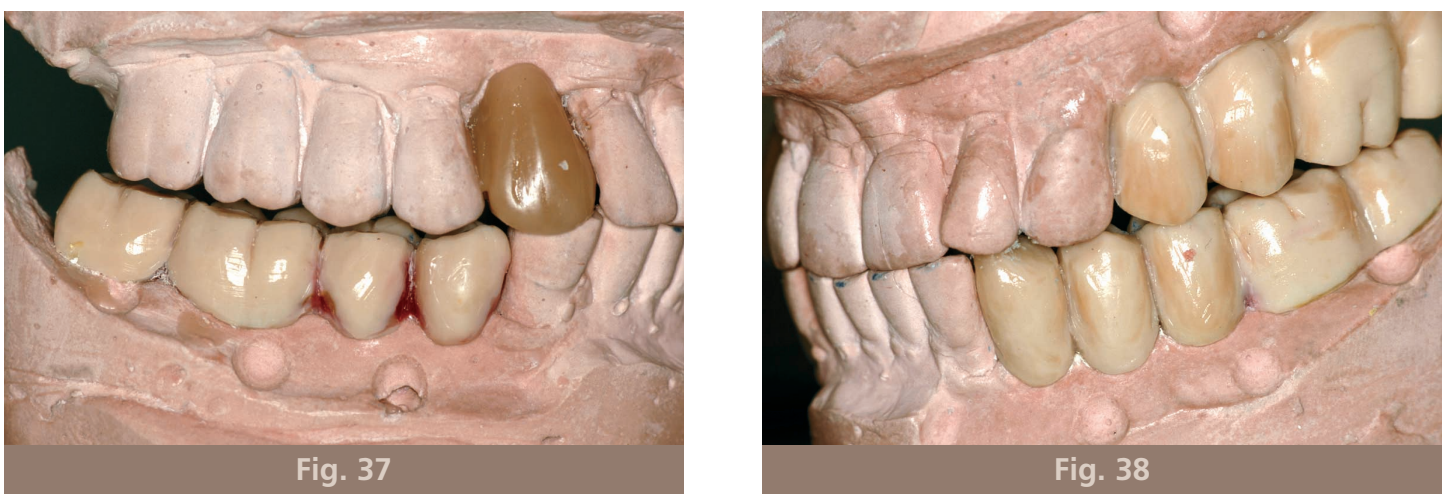

Fig. 37, 38 Le choix du concept occlusal sera celui de la fonction canine, chaque canine implantée faisant face à une canine naturelle riche en récepteurs desmodontaux. 


\section{Conclusion}

La connaissance de la neurophysiologie de l'appareil manducateur, basé en grande partie sur la présence de récepteurs desmodontaux, permet, en prothèse sur implant, d'orienter le choix du concept occlusal le mieux adapté au cas clinique considéré, en particulier lorsque une ou des canines sont remplacées par des implants.

\section{Ouvrages à consulter}

Bert M.

Les implants dentaires.

CdP édit, Paris, 1987.

Mamo $\mathrm{H}$.

La douleur.

Baillière édit, Paris, 1968.

Noordenbos W.

Pain.

Elsevier édit, Amsterdam, 1959.
Wall PD, Melzach R.

Pain mechanism,

a new theory.

Science 1965;150:971-979.

Woda A, Fontenelle A.

Organisation générale

du système nerveux.

In Chateau M.: ODF.

Julien Prélat édit, Paris, 1975.
Woda A.

Abrégé de physiologie

oro-faciale.

Masson édit, Paris, 1983.

Wolff J.

Das Gesetz

der Transformation

der Knochen.

Hirschwald édit, Berlin, 1892.

\section{SUMMARY}

\section{The canine tooth in implantology}

Marc BERT

\section{Keywords \\ - implant \\ - occlusion \\ - neurophysiology \\ - canine}

The replacement of a canine tooth by an implant will, in its neurophysiologic consequences, exchange a protection system based on the presence of periodontal membrane receptors with one that depends on an implant ankylosed in bone that can transmit only feeble information to be filtered by sensitive superior ganglia. The presence or absence of antagonistic receptors will guide the practitioner in selecting which occlusal concept to apply, with the first rule being that the simpler the concept, the more successfully it can be accomplished. 